\title{
M-TIMED PETRI NETS AND MARKOV CHAINS IN MODELLING OF COMPUTER SYSTEMS
}

\author{
W.M. Zuberek \\ Department of Computer Science, Memorial University \\ St.John's, NL, Canada A1B 3X5
}

\begin{abstract}
It is shown that the behavior of enhanced free-choice bounded M-timed Petri nets, i.e., Petri nets with two classes of transitions, immediate and timed ones, and with exponentially distributed firing times of timed transitions, can be represented by state-transition graphs which are finite continuous-time homogeneous Markov chains. Moreover, for each finite continuous-time homogeneous Markov chain there exists an enhanced free-choice bounded M-timed Petri net with the state-transition graph isomorphic to this chain. These two classes of models are thus equivalent. A straightforward application of (enhanced) M-timed Petri nets is modelling and performance evaluation of Markovian queueing systems, and in particular closed-network models of computer systems. Simple models of interactive systems are used as an illustration of modelling.
\end{abstract}

\section{INTRODUCTION}

A Petri net $[1,3,9,10]$ is an abstract, formal model of systems of events in which it is possible for some events to occur concurrently but there are constraints on the concurrence, precedence, or frequency of these occurrences $[4,9]$. A Petri net, however, is not complete enough for the study of systems performance since no assumption is made on the duration of systems activities. Several different concepts of "timed" Petri nets $[10,11,12,13]$ and stochastic Petri nets $[2,8]$ have been proposed by assigning deterministic or stochastic firing and/or enabling times to the transitions or places of Petri nets.

This paper describes an extension of the approach originated by Ramchandani [10] in which the "firing times" are assigned to transitions of Petri nets. In enhanced M-timed Petri nets, similarly as in generalized stochastic Petri nets [2], the set of transitions is subdivided into two disjoint classes of timed and immediate transitions, and the rates of exponentially distributed firing times are assigned to timed transitions only (immediate transitions fire instantaneously or in "zero time"). In M-timed nets, however, the concept of states is derived in a completely different way than in stochastic Petri nets $[2,8]$ which removes several restrictions of the stochastic approach [13]. It also provides a simple equivalence of finite continuous-time homogeneous Markov chains and a class of enhanced free-choice bounded M-timed Petri nets discussed in this paper.

This paper is organized in 6 main sections. Section 2 contains definitions of basic concepts for marked Petri nets. Enhanced Petri nets are introduced in Section 3 and M-timed Petri nets in Section 4. Section 5 shows equivalence of finite continuous-time homogeneous Markov chains and enhanced free-choice bounded M-timed Petri nets. The concept of equivalent M-timed nets is discussed in Section 6 .

\section{MARKED PETRI NETS}

A basic Petri net $[1,3,9] \mathbf{N}$ is a triple $\mathbf{N}=(P, T, A)$ where

$P$ is a finite, nonempty set of places,

$T$ is a finite, nonempty set of transitions,

$A$ is a set of directed arcs which connect places with transitions and transitions with places, $A \subset P \times T \cup T \times P$, such that for each transition there exists (at least one) arc directed to and (at least one) arc directed from it:

$$
\forall t \in T \exists p_{i}, p_{j} \in P:\left(p_{i}, t\right) \in A \wedge\left(t, p_{j}\right) \in A .
$$

A place $p$ is an input (or an output) place of a transition $t$ iff there exists an arc $(p, t)$ (or $(t, p)$, respectively) in the set $A$. The sets of all input and output places of a transition $t$ are denoted by $\operatorname{Inp}(t)$ and $O u t(t)$, respectively. Similarly, the sets of input and output transitions of a place $p$ are denoted by $\operatorname{Inp}(p)$ and $\operatorname{Out}(p)$. The notation is extended in an obvious way to sets of places and transitions, e.g., $\operatorname{Inp}\left(P_{i}\right)$, $\operatorname{Out}\left(T_{j}\right)$, etc.

A place $p$ is shared iff it is an input place for more that one transition. If a net does not contain shared places, it is conflict-free. A shared place $p$ is free-choice (or extended free-choice [3]) iff the input sets of all transitions sharing $p$ are identical. A net is free-choice iff all its shared places are free-choice.

In free-choice nets the relation of "sharing a place" is an equivalence relation in the set of transitions $T$, hence it determines a partition of $T$ into a set of free-choice equivalence classes denoted by $\operatorname{Free}(T)=\left\{T_{1}, T_{2}, \ldots, T_{k}\right\}$.

A net $\mathbf{N}_{i}=\left(P_{i}, T_{i}, A_{i}\right)$ is a $T_{i}$-implied subnet of a net $\mathbf{N}=(P, T, A)$ iff

(1) $T_{i} \subseteq T$,

(2) $A_{i}=A \cap\left(P \times T_{i} \cup T_{i} \times P\right)$,

(3) $P_{i}=\operatorname{Inp}\left(T_{i}\right) \cup \operatorname{Out}\left(T_{i}\right)$.

A marked Petri net $\mathbf{M}$ is a pair $\mathbf{M}=\left(\mathbf{N}, m_{0}\right)$ where

$\mathbf{N}$ is a basic Petri net $\mathbf{N}=(P, T, A)$,

$m_{0}$ is the initial marking function which assigns a nonnegative number of so called tokens to each place of the net, $m_{0}: P \rightarrow\{0,1, \ldots\}$.

Let any function $m: P \rightarrow\{0,1, \ldots\}$ be called a marking of a net $\mathbf{N}$.

A transition $t$ is enabled by a marking $m$ iff every input place $p \in \operatorname{In} p(t)$ contains at least one token, $m(p)>0$. The set of all transitions enables by a marking $m$ in a net $\mathbf{N}$ is denoted by $T(m)$.

Every transition enabled by a marking $m$ can fire. When a transition fires, a token is removed from each of its input places and a token is added to each of its output places. This 
determines a new marking in a net, a new set of enabled transitions, and so on.

A marking $m_{j}$ is directly reachable from a marking $m_{i}$ in a net $\mathbf{N}$ iff there exists a transition $t$ enabled by the marking $m_{i}, t \in T\left(m_{i}\right)$, such that:

$\forall p \in P: m_{j}(p)= \begin{cases}m_{i}(p)-1, & \text { if } p \in \operatorname{Inp}(t)-\operatorname{Out}(t), \\ m_{i}(p)+1, & \text { if } p \in \operatorname{Out}(t)-\operatorname{Inp}(t), \\ m_{i}(p), & \text { otherwise. }\end{cases}$

A firing sequence of a marking $m$ is any sequence of transitions $\left(t_{i_{1}}, t_{i_{2}}, \ldots\right)$ such that $m_{i_{0}}=m$, and for $\ell=1,2, \ldots$, $t_{i_{\ell}} \in T\left(m_{i_{\ell-1}}\right)$, and $m_{i_{\ell}}$ is directly reachable from $m_{i_{\ell-1}}$ by firing $t_{i_{\ell}}$.

A marking $m_{j}$ is (generally) reachable from a marking $m_{i}$ in a net $\mathbf{N}$ iff there exists a firing sequence which transforms $m_{i}$ into $m_{j}$.

A set $M(\mathbf{M})$ of reachable markings of a Petri net $\mathbf{M}$ is the set of all markings which are reachable from the initial marking $m_{0}$ (including $m_{0}$ ).

A marked Petri net $\mathbf{M}$ is bounded iff there exists a positive integer $k$ such that each marking in the set $M(\mathbf{M})$ assigns at most $k$ tokens to each place of the net

$$
\exists k>0 \forall m \in M(\mathbf{M}) \forall p \in P: m(p) \leq k .
$$

If a net $\mathbf{M}$ is bounded, its reachability set $M(\mathbf{M})$ is finite. Only bounded Petri nets are considered in this paper.

A reachability graph $\mathbf{G}$ of a net $\mathbf{M}$ is a directed graph $\mathbf{G}(\mathbf{M})=(V, D)$ where:

$V$ is a set of vertices which is equal to the set of reachable markings of the net $\mathbf{M}, V=M(\mathbf{M})$,

$D$ is a set of directed arcs, $D \subset V \times V$, such that $\left(m_{i}, m_{j}\right)$ is in $D$ iff $m_{j}$ is directly reachable from $m_{i}$ in $\mathbf{M}$.

A basic Petri net $\mathbf{N}$ is straight if for any initial marking $m_{0}$ its reachability graph is acyclic. If a net is straight, all its firing sequences are finite, and the net is bounded. The concept of straight nets is used in the next section in the definition of enhanced nets to eliminate infinite firing sequences in "immediate subnets".

\section{ENHANCED PETRI NETS}

Enhanced Petri nets have fwo disjoined classes of transitions, called immediate transitions and timed transitions. So, an enhanced Petri net $\mathbf{H}$ is a quadruple $\mathbf{H}=$ $\left(P, T_{t}, T_{0}, A\right)$ where

$\left(P, T_{t} \cup T_{0}, A\right)$ is a basic Petri net,

$T_{t}$ is a set of timed transitions such that

(1) $\operatorname{Out}\left(\operatorname{Inp}\left(T_{t}\right)\right)=T_{t}$, which means that shared places can be shared only by immediate transitions or only by timed transitions, but not by both classes;

(2) $\forall t \in T_{t}: \#(\operatorname{Inp}(t))=\#($ Out $(t))=1$

where $\#(X)$ denotes the number of elements in the set $X$; since each timed transition can have only one input and one output place, these single places are denoted by inp $(t)$ and out $(t)$;

$T_{0}$ is a set of immediate transitions such that the $\left(T_{0}\right)$ implied subnet of $\left(P, T_{t} \cup T_{0}, A\right)$ is straight, and the sets $T_{t}$ and $T_{0}$ are disjoint.
The total set of timed and immediate transitions is denoted by $T=T_{t} \cup T_{0}$.

A marked (enhanced) Petri net $\mathbf{M}$ is a pair $\mathbf{M}=\left(\mathbf{H}, m_{0}\right)$ where

$\mathbf{H}$ is an enhanced Petri net $\mathbf{H}=\left(P, T_{t}, T_{0}, A\right)$, $m_{0}$ is the intial marking function, $m_{0}: P \rightarrow\{0,1, \ldots\}$.

The set of all transitions enabled by a marking $m$ is denoted by $T(m)$, as before. Moreover, $T_{t}(m)$ denotes the set of timed transitions enabled by $m$, and $T_{0}(m)$ the set of immediate transitions enabled by $m$.

An enhance function of a marking $m$ in an enhanced net $\mathbf{H}$ is any function $h: T \rightarrow\{0,1, \ldots\}$ such that

(1) there exists a finite (possibly empty) firing sequence of immediate transitions $\left(t_{i_{1}}, t_{i_{2}}, \ldots, t_{i_{k}}\right)$ which transforms the marking $m$ into $m_{k}$, and the set of immediate transitions enabled by $m_{k}, T_{0}\left(m_{k}\right)$, is empty, and

(2) for each immediate transition $t \in T_{0}$ the number of occurrences of $t$ in this firing sequence is equal to $h(t)$, while for each timed transition $t \in T_{t}, h(t)=0$.

The set of all enhance functions of a marking $m$ is denoted by $\operatorname{Enh}(m)$. Since the $T_{0}$-implied subnet of an enhanced net $\mathbf{H}$ is straight, all firing sequences of immediate transitions are finite, and for each marking $m$ the set $\operatorname{Enh}(m)$ is also finite.

Moreover, a selection function of a marking $m$ in an enhanced net $\mathbf{H}$ is any function $g: T \rightarrow\{0,1, \ldots\}$ such that

$$
\forall t \in \operatorname{Inp}\left(T_{t}\right): m(p)=\sum_{t \in \text { Out }(p)} g(t)
$$

and for each immediate transition $t \in T_{0}, g(t)=0$, i.e., any function which describes all possible simultaneous firings of enabled timed transitions (and some transitions may fire "several times"). The set of all selection functions of a marking $m$ is denoted by $\operatorname{Sel}(m)$. It should be observed that if the input places of timed transitions are conflict-free, there is only one selection function such that

$$
\forall t \in T_{t} \quad: \quad g(t)=m(\operatorname{inp}(t))
$$

where $\operatorname{inp}(t)$ denotes the single input place of $t$.

If timed transitions belong to free-choice classes of transitions, the set $\operatorname{Sel}(\mathrm{m})$ describes all possible "free choices" of firing transitions. Enhance and selection functions are used in the description of timed nets.

Example 1. For the enhanced free-choice Petri net shown in Fig.1 (as usual, places are denoted by circles, immediate transitions by bars, timed transitions by rectangles, and the initial marking is represented by dots inside places), the set of enhance functions, $\operatorname{Enh}\left(m_{0}\right)$, contains only one function, $\operatorname{Enh}\left(m_{0}\right)=\{[0,0,0,0,1,1]\}$, which corresponds to the firing sequence $\left(t_{6}, t_{5}\right)$. This enhance function transforms the marking $m_{0}$ into $m_{1}=[0,1,0,0,1,0]$, and the set of selection functions of $m_{1}, \operatorname{Sel}\left(m_{1}\right)$, contains two functions (the place $p_{5}$ is a free-choice place) $\operatorname{Sel}\left(m_{1}\right)=$ $\{[1,0,1,0,0,0],[0,1,1,0,0,0]\}$.

\section{ENHANCED M-TIMED PETRI NETS}

In timed Petri nets, each transition $t$ takes a "real" time to fire, i.e., there is a "firing time" function which determines 


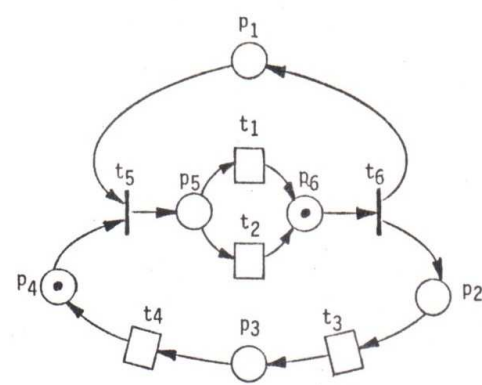

Fig.1.Enhanced free-choie Petri net $\mathbf{M}$.

the duration of firing for each transitions of a net. When a transition $t$ is enabled, a firing can be initiated by removing one token from all $t$ 's input places. This token remains in the transition $t$ for the "firing time", and then the firing terminates by adding a single token to each of $t$ 's output places. Each of the firings is initiated in the same instant of time in which it is enabled. If a transition is enabled while it fires, a new, independent firing can be initiated. For conflictfree nets, all enabled transitions immediately initiate their firings. If a net contains conflicts, the selection of transitions for firing is assumed to be a random process which can be described by corresponding probabilities.

The states of timed Petri nets correspond to net "configurations" between consecutive initiations and terminations of firings. The description of states must take into account the distribution of tokens in places as well as in firing transitions. Both distributions can be described by a pair of functions which map the set of places (the marking function) and the set of transitions (the firing function) into the set of nonnegative integers.

In enhanced timed Petri nets, the immediate transitions fire in zero time, i.e., they do not contribute to "delays", while the timed transitions require a positive time to fire. Since all transitions (immediate and timed) initiate their firings in the same instants of time in which they are enabled, and immediate transitions also immediately terminate their firings, it is convenient to consider consecutive state-transitions as composed of 3 consecutive steps: (1) termination of a timed transition firing, (2) a sequence (possibly empty) of immediate transition firings which terminates when no immediate transition is enabled (this sequence is described by an enhance function $h$ ), (3) initiation of new timed transition firings (which is described by a selection function $g$ ). All 3 steps take place in the same instant of time.

The firing times of (timed) transitions can be described in several ways. In D-timed Petri nets $[10,11,12]$ they are deterministic (or constant), i.e., there is a positive (rational) number assigned to each (timed) transition of a net which determines the duration of transition's firings. In M-timed Petri nets (or stochastic Petri nets) [2,8], the firing times are exponentially distributed random variables, and the corresponding firing rates are assigned to (timed) transitions of a net. The memoryless property of the exponential distribution is the basic factor in analysis of M-timed Petri nets.

An enhanced M-timed Petri net $\mathbf{T}$ is a triple $\mathbf{T}=(\mathbf{M}, c, r)$ where:

$\mathbf{M}$ is a marked enhanced free-choice bounded Petri net,
$\mathbf{M}=\left(\mathbf{H}, m_{0}\right), \mathbf{H}=\left(P, T_{t}, T_{0}, A\right)$,

$c$ is a choice function which assigns a free-choice probability to each transition of a net in such a way that

$$
\forall T_{i} \in \operatorname{Free}(T): \sum_{t \in T_{i}} c(t)=1
$$

$r$ is a firing rate function which assigns a positive real number $r(t)$ to each timed transition $t$ of the net, $r: T_{t} \rightarrow \mathbf{R}^{+}$, and $\mathbf{R}^{+}$denotes the set of positive real numbers; the firing time of a timed transition $t$ is a random variable $v(t)$ with the distribution function

$$
\operatorname{Prob}(v(t)>\tau)=e^{-\tau * r(t)}, \quad \tau>0
$$

A state $s$ of an M-timed Petri net $\mathbf{T}$ is a pair of functions $s=(m, f)$ where:

$m$ is a marking function, $m: P \rightarrow\{0,1, \ldots\}$,

$f$ is a firing function which indicates (for timed transitions) the numbers of active firings, i.e., the numbers of firings which have been initiated but are not yet terminated, $f: T_{t} \rightarrow\{0,1, \ldots\}$.

An initial state $s_{i}$ of a free-choice net $\mathbf{T}$ is a pair $s_{i}=$ $\left(m_{i}, f_{i}\right)$ where $f_{i}$ is a selection function from the set $\operatorname{Sel}\left(m_{0 i}\right)$, $f_{i} \in \operatorname{Sel}\left(m_{0 i}\right)$, and the (intermediate) marking $m_{0 i}$ is determined by an enhance function $h_{\ell}$ from the set $\operatorname{Enh}\left(m_{0}\right)$, $h_{\ell} \in \operatorname{Enh}\left(m_{0}\right)$, in the following way

$\forall p \in P: m_{0 i}(p)=m_{0}(p)+\sum_{t \in \operatorname{Inp}(p)} h_{\ell}(t)-\sum_{t \in \text { Out }(p)} h_{\ell}(t)$,
$\forall p \in P: m_{i}(p)=m_{o i}(p)-\sum_{t \in \text { Out }(p)} f_{i}(t)$.

A free-choice net $\mathbf{T}$ may have several different initial states.

A state $s_{j}=\left(m_{j}, f_{j}\right)$ is directly reahable (or $\left(t_{k}, h_{\ell}, g_{n}\right)$ reachable) from the state $s_{i}=\left(m_{i}, f_{i}\right)$ iff:

(1) $f_{i}\left(t_{k}\right)>0$

(2) $h_{\ell} \in \operatorname{Enh}\left(m_{i k}\right)$,

(3) $\forall p \in P: m_{i k}(p)=m_{i}(p)+ \begin{cases}1, & \text { if } p \in O u t\left(t_{k}\right), \\ 0, & \text { otherwise, }\end{cases}$

(4) $g_{n} \in \operatorname{Sel}\left(m_{i k l}\right)$,

(5) $\forall p \in P: m_{i k l}(p)=$ $m_{i k}(p)+\sum_{t \in \operatorname{Inp}(p)} h_{\ell}(t)-\sum_{t \in O u t(p)} h_{\ell}(t)$,

(6) $\forall p \in P: m_{j}(p)=m_{i k l}(p)-\sum_{t \in O u t(p)} g_{n}(t)$,

(7) $\forall t \in T_{t}: f_{j}(t)=f_{i}(t)+g_{n}(t)- \begin{cases}1, & \text { if } t=t_{k}, \\ 0, & \text { otherwise }\end{cases}$

The state $s_{j}$ which is $\left(t_{k}, h_{\ell}, g_{n}\right)$-reachable from the state $s_{i}$ is thus obtained by the termination of a $t_{k}$ firing (1), updating the marking of a net (3), performing all immediate firings (if any) which are indicated by the enhance function $h_{\ell}$ from the set $\operatorname{Enh}\left(m_{i k}\right)(2)$, and which creates an intermediate marking $m_{i k l}(5)$, and then initiating new timed firings (if any) which are enabled by $m_{i k l}(4,6$, and 7 ).

Similarly as for reachable markings, a state $s_{j}$ is (generally) reachable from a state $s_{i}$ if there is a sequence of directly reachable states from the state $s_{i}$ to the state $s_{j}$. Also, a set $S(\mathbf{T})$ of reachable states is defined as the set 
of all states of a net $\mathbf{T}$ which are reachable from the initial states (including the initial states). For enhanced free-choice bounded M-timed nets the sets of reachable states are finite.

A state-transition graph $\mathbf{G}$ of an M-timed Petri net $\mathbf{T}$ is a labeled directed graph $\mathbf{G}(\mathbf{T})=(V, D, u)$ where:

$V$ is a set of vertices which is equal to the set of reachable states of the net $\mathbf{T}, V=S(\mathbf{T})$,

$D$ is a set of directed arcs, $D \subset V \times V$, such that $\left(s_{i}, s_{j}\right)$ is in $D$ iff $s_{j}$ is directly reachable from $s_{i}$ in $\mathbf{T}$,

$u$ is a labeling function which assigns the rate of transitions from $s_{i}$ to $s_{j}$ to each arc $\left(s_{i}, s_{j}\right)$ in the set $D, u: D \rightarrow$ $\mathbf{R}^{+}$, in such a way that if $s_{j}$ is $\left(t_{k}, h_{\ell}, g_{n}\right)$-reachable from $s_{i}=\left(m_{i}, f_{i}\right)$, then

$$
u\left(s_{i}, s_{j}\right)=r\left(t_{k}\right) * f_{i}\left(t_{k}\right) * \prod_{t \in T} c(t)^{h_{\ell}(t)+g_{n}(t)} .
$$

Example 2. For the enhanced free-choice M-timed Petri net $\mathbf{T}_{1}$ shown in Fig.2 (the firing-rate and the choice functions are given as additional descriptions of transitions), the derivation of the set $S(\mathbf{T})$ of reachable states and the transition rate function $u$ are shown in Tab.1.

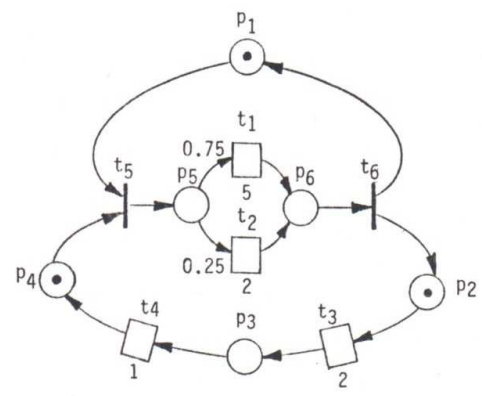

Fig.2. M-timed Petri net $\mathbf{T}_{1}$.

\begin{tabular}{|c|c|c|c|c|c|c|}
\hline$s_{i}$ & $\begin{array}{ll} & m_{i} \\
123456 & \end{array}$ & $\begin{array}{c}f_{i} \\
1234\end{array}$ & $t_{k}$ & $\begin{array}{l}h_{\ell} \\
56\end{array}$ & $\begin{array}{c}g_{n} \\
1234\end{array}$ & $s_{j}$ \\
\hline \multirow[t]{2}{*}{1} & $\begin{array}{lllllll}0 & 0 & 0 & 0 & 0 & 0\end{array}$ & 01110 & 2 & 01 & 001010 & 3 \\
\hline & & & 3 & 00 & $\begin{array}{lllll}0 & 0 & 0 & 1\end{array}$ & 4 \\
\hline \multirow[t]{2}{*}{2} & $\begin{array}{llllll}0 & 0 & 0 & 0 & 0 & 0\end{array}$ & 1010 & 1 & 01 & $\begin{array}{llll}0 & 0 & 1 & 0\end{array}$ & 3 \\
\hline & & & 3 & 00 & $\begin{array}{llll}0 & 0 & 0 & 1\end{array}$ & 5 \\
\hline 3 & 10000000 & 0020 & 3 & 00 & $\begin{array}{llll}0 & 0 & 0 & 1\end{array}$ & 6 \\
\hline \multirow[t]{2}{*}{4} & 00000000 & $\begin{array}{llll}0 & 101\end{array}$ & 2 & 01 & 00010 & 6 \\
\hline & & & 4 & 00 & 0000 & 7 \\
\hline \multirow[t]{2}{*}{5} & $\begin{array}{llllll}0 & 0 & 0 & 0 & 0 & 0\end{array}$ & 1001 & 1 & 01 & $\begin{array}{llll}0 & 0 & 1 & 0\end{array}$ & 6 \\
\hline & & & 4 & 00 & 0000 & 8 \\
\hline \multirow[t]{3}{*}{6} & 1000000 & $\begin{array}{llll}0 & 0 & 1 & 1\end{array}$ & 3 & 00 & $\begin{array}{llll}0 & 0 & 0 & 1\end{array}$ & 9 \\
\hline & & & 4 & 10 & 0100 & 1 \\
\hline & & & & & 1000 & 2 \\
\hline \multirow[t]{2}{*}{7} & $\begin{array}{llllll}0 & 0 & 0 & 1 & 0 & 0\end{array}$ & 0100 & 2 & 11 & 01110 & 1 \\
\hline & & & & & 1010 & 2 \\
\hline \multirow[t]{2}{*}{8} & $\begin{array}{llllll}0 & 0 & 0 & 1 & 0 & 0\end{array}$ & 1000 & 1 & 11 & 01110 & 1 \\
\hline & & & & & 1010 & 2 \\
\hline \multirow[t]{2}{*}{9} & 10000000 & 0002 & 4 & 10 & 0100 & 4 \\
\hline & & & & & 1000 & 5 \\
\hline
\end{tabular}

Tab.1. The set of reachable states for $\mathbf{T}_{1}$.

\section{MARKOV CHAINS AND TIMED PETRI NETS}

A continuous-time homogeneous Markov chain can be defined [6] as a pair $\mathbf{Q}=(S, R)$ where

$S$ is a set of (discrete) states of the chain, and

$R$ is a square matrix of transition-rates between states from the set $S$, i.e., $R[i, j]$ denotes the rate of transitions from the state $s_{i}$ to the state $s_{j}$ (if $R[i, j]=0$, there are no direct transitions between $s_{i}$ and $s_{j}$ ).

If the set of states $S$ is finite, the corresponding Markov chain is also finite. From the definition of state-transition graphs of enhanced M-timed Petri nets immediately follows that the state-transition graph of an enhanced free-choice bounded M-timed Petri net is a finite continuous-time homogeneous Markov chain. Moreover, it can be shown that for each finite continuous-time homogeneous Markov chain there exists an enhanced M-timed Petri net such that its state-transition graph is isomorphic to the Markov chain.

A Petri net model of a finite Markov chain $\mathbf{Q}=(S, R)$ is an enhanced free-choice bounded M-timed Petri net $\mathbf{T}=$ $(\mathbf{M}, c, r), \mathbf{M}=\left(\mathbf{H}, m_{0}\right), \mathbf{H}=\left(P, T_{t}, T_{0}, A\right)$ where:

(1) $T_{t}=\left\{t_{i} \mid s_{i} /\right.$ in $\left.S\right\}$,

(2) $T_{0}=\left\{" t_{j, k} \mid R[j, k]>0\right\}$

(3) $P=\left\{p_{i}, \hat{p}_{i} \mid s_{i} \in S\right\}$,

(4) $A=\left\{\left(p_{i}, t_{i}\right),\left(t_{i}, \hat{p}_{i}\right) \mid t_{i} \in T_{t}\right\} \cup$ hfill $\left\{\left(\hat{p_{j}}, t j, k\right),\left(t j, k, p_{k}\right) \mid t_{j, k} \in T_{0}\right\}$,

(5) $\forall p_{j} \in P: m_{0}\left(p_{j}\right)= \begin{cases}1, & \text { if } j=1, \\ 0, & \text { otherwise, }\end{cases}$

(6) $\forall t_{j} \in T_{t}: r\left(t_{j}\right)=\sum_{1 \leq k \leq \#(S)} R[j, k]$,

(7) $\forall t \in T: c(t)= \begin{cases}1, & \text { if } t \in T_{t}, \\ R[j, k] / r\left(t_{j}\right), & \text { if } t=t_{j, k} .\end{cases}$

It can be observed that for each timed transition $t_{i}$ there is exactly one input and one output place, $p_{i}$ and $\hat{p}_{i}$, respectively, that all immediate transitions have exactly one input and one output place, which means that all shared places (only the places $\hat{p}_{i}$ may be shared) are free-choice, and that in each state of the net the number of firing transitions cannot be greater than the number of tokens assigned by the initial marking, i.e. 1 (in fact, at any instant of time, there is a single token in the modelling net which can be either "in" a firing timed transition, or in one of the places). The net implied by the set of immediate transitions is obviously straight because it is acyclic. Consequently, the states of the modelling Petri net directly correspond to the (firing) timed transitions (one timed transition per one state) and thus to the states of the modelled Markov chain, all free choices are restricted to single free-choice places (there is a single token in the net), and the probabilities of free-choice classes combined with the firing rates of timed transitions correspond to the rates of transitions between states of the modelled Markov chain. The state-transition graph of the modelling Petri net is thus isomorphic to the modelled Markov chain.

Example 3. For the Markov chain with the following matrix of transition rates

\begin{tabular}{|c|ccccc|}
\hline$R$ & 1 & 2 & 3 & 4 & 5 \\
\hline 1 & 0.0 & 4.0 & 0.0 & 0.0 & 0.0 \\
2 & 2.0 & 0.0 & 3.0 & 0.0 & 0.0 \\
3 & 0.0 & 2.0 & 0.0 & 2.0 & 0.0 \\
4 & 0.0 & 0.0 & 2.0 & 0.0 & 1.0 \\
5 & 0.0 & 0.0 & 0.0 & 2.0 & 0.0 \\
\hline
\end{tabular}


the modelling net is shown in Fig.3, and the derivation of its state-transition graph is given in Tab.2.

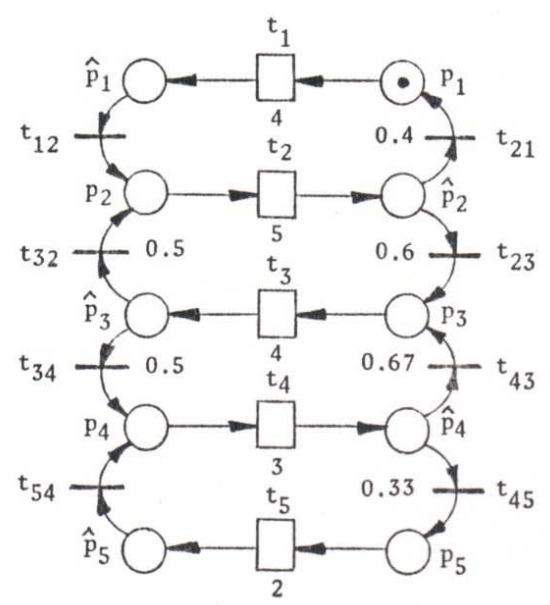

Fig.3. Cann0nical M-timed Petri net $\mathbf{T}_{2}$.

\begin{tabular}{|c|c|c|c|c|c|}
\hline$s_{i}$ & $\begin{aligned} & m_{i} \\
& 12345 \hat{1} \hat{2} \hat{3} \hat{4} \hat{5}\end{aligned}$ & $\begin{array}{c}f_{i} \\
12345\end{array}$ & $t_{k}$ & $s_{j}$ & $u\left(s_{i}, s_{j}\right)$ \\
\hline 1 & $\begin{array}{lllllllllll}0 & 0 & 0 & 0 & 0 & 0 & 0 & 0 & 0 & 0\end{array}$ & 100000 & 1 & 2 & 4.00 \\
\hline \multirow[t]{2}{*}{2} & $\begin{array}{llllllllll}0 & 0 & 0 & 0 & 0 & 0 & 0 & 0 & 0 & 0\end{array}$ & $\begin{array}{lllll}0 & 1 & 0 & 0 & 0\end{array}$ & 2 & 3 & 3.00 \\
\hline & & & & 1 & 2.00 \\
\hline \multirow[t]{2}{*}{3} & $\begin{array}{llllllllll}0 & 0 & 0 & 0 & 0 & 0 & 0 & 0 & 0 & 0\end{array}$ & $\begin{array}{llllll}0 & 0 & 1 & 0 & 0\end{array}$ & 3 & 4 & 2.00 \\
\hline & & & & 2 & 2.00 \\
\hline \multirow[t]{2}{*}{4} & $\begin{array}{llllllllll}0 & 0 & 0 & 0 & 0 & 0 & 0 & 0 & 0 & 0\end{array}$ & $\begin{array}{lllllllll}0 & 0 & 0 & 1 & 0\end{array}$ & 4 & 5 & 1.00 \\
\hline & & & & 3 & 2.00 \\
\hline 5 & $\begin{array}{llllllllll}0 & 0 & 0 & 0 & 0 & 0 & 0 & 0 & 0 & 0\end{array}$ & $\begin{array}{llllll}0 & 0 & 0 & 0 & 1\end{array}$ & 5 & 4 & 2.00 \\
\hline
\end{tabular}

Tab.2. The set of reachable states for $\mathbf{T}_{2}$.

It can be observed that the M-timed Petri net modelling the state graph $\mathbf{G}\left(\mathbf{T}_{1}\right)$ is quite different from the net $\mathbf{T}_{1}$ shown in Fig.2 (it contains 9 timed transitions, 18 places and 18 immediate transitions), but its behavior (i.e., the state-transition graph) is the same as that of $\mathbf{T}_{1}$.

\section{EQUIVALENT M-TIMED PETRI NETS}

Since the behavior of (enhanced) free-choice bounded Mtimed Petri nets can be represented by finite state-transition graphs, a very simple equivalence relation can be defined in the class of (not necessarily free-choice) bounded M-timed Petri nets. Two such nets are equivalent iff their statetransition graphs are isomorphic, i.e., iff there exists a oneto-one mapping which transforms one state-transition graph into another.

M-timed Petri nets models of finite Markov chains (as defined in section 5) are much simpler than the class of enhanced free-choice bounded M-timed Petri nets discussed in section 4 (the timed subnets are in fact conflict-free, and there are no simultaneous firings). Since such an equivalent modelling M-timed Petri net exists for each M-timed Petri net with finite state-transition graph, the modelling M-timed Petri nets are called the "canonical" representation of finitestate M-timed Petri nets. Canonical nets are rather large since the number of timed transitions is always equal to the number of states, the number of immediate transitions is equal to the number of directed arcs in the state-transition graphs, and the number of places is twice as large as the number of states. On the other hand, analysis of canonical representations is much simpler, and in particular, state graphs can be derived directly from canonical nets. The size of canonical nets is thus the "price" for their simplicity.

In many special cases the modelling nets can be much more compact than the canonical representation. For example, the transition-rate matrix of the net from Example 3 is bidiagonal, and the transition-rates $R[i+1, i]$ are constant, while $R[i, i+1]$ are linearly decreasing with $i, i=1, \ldots, 4$. This is the characteristic state-transition model of $M / M / 1 / / k$ systems [6,7], i.e., single server systems with a finite population $k(k=4$ in the example), and with exponentially distributed service and interarrival times. A typical example of such a system is a central server model of an interactive computer system $[5,6]$ with exponentially distributed terminal and service times, and with $k$ being the number of active users (or terminals). A compact and general Petri net model of $M / M / 1 / / k$ systems is shown in Fig.4 [14]; $t_{1}$ represents the central server and $p_{2}$ its queue of waiting jobs, $t_{2}$ models the terminals (or users), and the total number of initial tokens in $p_{2}, p_{3}, p_{4}$ and $p_{5}$ determines $k$. It should be observed that the net is conflict-free. The derivation of the set of reachable states for $\mathbf{T}_{3}$ is shown in Tab.3.

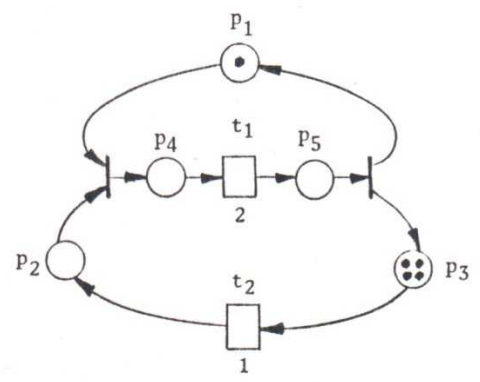

Fig.4. M-timed Petri net $\mathbf{T}_{3}$.

\begin{tabular}{|c|c|c|c|c|c|}
\hline$s_{i}$ & $\begin{array}{c}m_{i} \\
12345\end{array}$ & $\begin{array}{c}f_{i} \\
12\end{array}$ & $t_{k}$ & $s_{j}$ & $u\left(s_{i}, s_{j}\right)$ \\
\hline 1 & 1000000 & 04 & 2 & 2 & 4.00 \\
\hline \multirow[t]{2}{*}{2} & $\begin{array}{llllll}0 & 0 & 0 & 0 & 0\end{array}$ & 13 & 1 & 1 & 2.00 \\
\hline & & & 2 & 3 & 3.00 \\
\hline \multirow[t]{2}{*}{3} & $\begin{array}{lllll}0 & 1 & 0 & 0 & 0\end{array}$ & 12 & 1 & 2 & 2.00 \\
\hline & & & 2 & 4 & 2.00 \\
\hline \multirow[t]{2}{*}{4} & 02000 & 11 & 1 & 3 & 2.00 \\
\hline & & & 2 & 5 & 1.00 \\
\hline 5 & 03000 & 10 & 1 & 4 & 2.00 \\
\hline
\end{tabular}

Tab.3. The set of reachable states for $\mathbf{T}_{3}$.

In fact, the net from Fig. 4 is even more general and it can model the $M / M / n / / k$ systems where the $n$ parameter (i.e., the number of central server's channels) corresponds to the total number of initial tokens in $p_{1}, p_{4}$ and $p_{5}$.

The M-timed Petri net from Fig.2 is a model of another interactive central server system in which the service times have a two-stage hyperexponential distribution $\left(t_{1}\right.$ and $\left.t_{2}\right)$, while the terminal times have a two-stage hypoexponential distribution ( $t_{3}$ and The net $\mathbf{T}_{1}$ is thus a model of $E_{2} / H_{2} / n / / k$ systems, with $n=1$ and $k=2$ (Tab.1). 
Many performance measures can be obtained from stationary (or equilibrium) probabilities of the states $[6,7]$. For finite-state systems the stationary probabilities $x(s)$ of the states $s \in S(\mathbf{T})$ are obtained as the solution of the following system of linear equations $[5,6]$

$$
\begin{aligned}
& \sum_{1 \leq j \leq N} u\left(s_{j}, s_{i}\right) * x\left(s_{j}\right)=x\left(s_{i}\right) * \sum_{1 \leq j \leq N} u\left(s_{i}, s_{j}\right) \\
& i=1, \ldots,
\end{aligned}
$$

where $N$ is the number of states in the set $S(\mathbf{T})$.

For the net $\mathbf{T}_{1}$ (Fig.2, Tab.1), the stationary probabilities of the stases are given in Tab.4.

\begin{tabular}{|c|c|}
\hline$s_{i}$ & $x\left(s_{i}\right)$ \\
\hline 1 & 0.030 \\
2 & 0.051 \\
3 & 0.079 \\
4 & 0.071 \\
5 & 0.094 \\
6 & 0.310 \\
7 & 0.036 \\
8 & 0.019 \\
9 & 0.310 \\
\hline
\end{tabular}

Tab.4. Stationary probabilities for $\mathbf{T}_{1}$.

Since the server is idle in the states $s_{3}, s_{6}$ and $s_{9}\left(m_{3}\left(p_{1}\right)=\right.$ $m_{6}\left(p_{1}\right)=m_{9}\left(p_{1}\right)=1$ ) (Tab.1), the stationary probability that the system is idle is equal to $x\left(s_{3}\right)+x\left(s_{6}\right)+x\left(s_{9}\right)=0.699$ (Tab.4). Then the utilization of the system is immediately $1-0.699=0.301$. Since the average service time is equal to $c\left(t_{1}\right) / r\left(t_{1}\right)+c\left(t_{2}\right) / r\left(t_{2}\right)=0.275$ time units, the average throughput rate is $0.301 / 0.275=1.095$ jobs per time unit. Since this throughput rate corresponds to 2 jobs $(k=2)$, the average turnaround time is equal to $2 / 1.095=1.827$ time units, and is composed of the average terminal time $\left(1 / r\left(t_{3}\right)+1 / r\left(t_{4}\right)=1.5\right.$ time units), average service time (0.275 time units) and the average waiting time equal to $1.827-1.5-0.275=0.052$ time units.

Many other results can be derived in a very similar way.

\section{CONCLUDING REMARKS}

It has been shown that the behavior of enhanced freechoice bounded M-timed Petri nets can be represented by equivalent finite continuous-time Markov chains. Moreover, for each finite continuous-time Markov chains there exists a simple enhanced free-choice bounded M-timed Petri net such that its state-transition graph is isomorphic to the Markov chain. Consequently, these two classes of models are equivalent. Also, a simple equivalence relation for bounded Mtimed Petri nets is proposed, and some examples of equivalent models of interactive computer systems are shown. .sp0.5 M-timed Petri net models are usually rather simple, and their parameters correspond in a natural way to components or activities of the modelled systems (e.g., the number of users, the number of server's channels, etc.). The state space can be generated automatically from model specifications, and the stationary probabilities of the states (which are the basis for many performance measures, such as utilization factors, turnaround times, throughput rates) can be obtained from state-transition graphs by known techniques used for analysis of Markov chains [5,6,7]. .sp0.5 The class of enhanced timed Petri nets discussed in this paper is restricted in several ways (free-choice bounded nets), some of the restrictions, however, can be removed easily by appropriate extensions of the formalism. In fact, Petri nets with more general conflicts (or "random switches" [2]) and with inhibitor arcs [14] can be handled in a very similar way provided the probabilities of conflicting firings are known and included in the state description. Also, unbounded nets (with infinite sets of reachability states) can be analyzed using recursive formulas which reduce infinite sets of states to their finite behavioral equivalents.

\section{REFERENCES}

1. T. Agerwala, Putting Petri nets to work; IEEE Computer Magazine, vol.12, no.12, pp.85-94, 1979.

2. M. Ajmone Marsan, G. Conte, G. Balbo, A class of generalized stochastic Petri nets for the performance evaluation of multiprocessor systems; ACM Trans. on Computer Systems, vol.2, no.2, pp.93-122, 1984.

3. W. Brauer (ed.), Net theory and applications; Proc. of the Advanced Course on General Net Theory of Processes and Systems, Hamburg 1979; Lecture Notes in Computer Science 84, Springer Verlag 1980.

4. M. Diaz, Modeling and analysis of communication and cooperation protocols using Petri net based models; Computer Networks, vol.6, no.6, pp.419-441, 1982.

5. E. Gelenbe, I. Mitrani, Analysis and synthesis of computer systems; Academic Press 1980.

6. L. Kleinrock, Queueing systems, vol.1: Theory, vol.2: Computer applications; J. Wiley \& Sons 1975, 1976.

7. H. Kobayashi, Modeling and analysis - an introduction to system performance evaluation methodology; Addison-Wesley 1981.

8. M.K. Molloy, Performance analysis using stochastic Petri nets; IEEE Trans. on Computers, vol.31, no.9, pp.913917, 1982.

9. J.L. Peterson, Petri net theory and the modeling of systems, Prentice-Hall 1981.

10. C. Ramchandani, Analysis of asynchronous concurrent systems by timed Petri nets; Project MAC Technical Report MAC-TR-120, Massachusetts Institute of Technology, Cambridge MA, 1974.

11. R.R. Razouk, The derivation of performance expressions for communication protocols from timed Petri nets; Computer Communication Review, vol.14, no.2, pp.210-217, 1984. item[] 12. J. Sifakis, Use of Petri nets for performance evaluation; in: Measuring, modelling and evaluating computer systems, pp.75-93, North-Holland 1977.

13. W.M. Zuberek, Performance evaluation using extended timed Petri nets; Proc. Int. Workshop on Timed Petri Nets, Torino, Italy, 1985.

14. W.M. Zuberek, Enhanced M-timed Petri nets, modelling and performance evaluation; Technical Report \#8514, Department of Computer Science, Memorial University of Newfoundland, St. John's, Canada A1C 5S7, 1985. 